21st Particles and Nuclei International Conference (PANIC 2017)

International Journal of Modern Physics: Conference Series

Vol. 46 (2018) 1860078 (6 pages)

(C) The Author(s)

DOI: $10.1142 / \mathrm{S} 2010194518600789$

\title{
The Upgrade of Endcap TOF in BESIII
}

\author{
Zhi $\mathrm{Wu}$ (for the BESIII Endcap-TOF group) \\ Institute of High Energy Physics, Chinese Academy of Sciences, Beijing 100049, China \\ State Key Laboratory of Particle Detection and Electronics, Beijing 100049, China \\ wuz@ihep.ac.cn
}

Published 3 May 2018

\begin{abstract}
In this paper the new endcap Time-of-Flight system (ETOF) of BESIII experiment at the IHEP is described. The new detector module is composed of multi-gap resistive plate chamber (MRPC) with the gas mixture $90 \%$ Freon $+5 \% \mathrm{~S} F_{6}+5 \%$ iso- $C_{4} H_{10}$. The whole system including the detectors, the gas system, the high voltage (HV) system, the readout electronics system is described. Finally the installation and commissioning of the whole system is introduced briefly. After the calibration with Bhabha events, the very promising time resolutions of 69 and 60 ps for one-end and two-ends of MRPCs are obtained. The average detection efficiency of two-ends is about $97.5 \%$.
\end{abstract}

Keywords: BESIII; MRPC; upgrade of ETOF.

\section{Introduction}

The BESIII experiment ${ }^{1}$ studies $e^{+}-e^{-}$collisions in the $\tau$-charm energy region at the Beijing Electron and Positron Collider II (BEPCII) ${ }^{2}$. The BESIII detector consists of a high precision drift chamber, Time-Of-Flight counters (TOF), a $1 \mathrm{~T}$ superconducting solenoid magnet, a CsI crystal Electromagnetic Calorimeter (EMC) and a muon identifier chamber (MUC). The original endcap time-of-flight (ETOF) particle identification (PID) system of the Beijing Spectrometer III (BESIII) consists of two disks of 48 pieces of plastic scintillating counters covering the polar angle region of $0.83<|\cos \theta|<0.96$ as shown in Fig. 1. Each counter consists of fast scintillators block (BC204) readout by fine-mesh photomultiplier tubes from the inner end. The time resolution measured by ETOF detector is $138 \mathrm{ps}$ for $\pi$ 's, which cannot completely satisfy the higher precision requirement of physics ${ }^{3}$, while the time resolution of BTOF is $70 \mathrm{ps}$ for $\pi$ 's. One significant reason that can worsen the ETOF resolution is the multiple scattering of particles in the thick aluminium

This is an Open Access article published by World Scientific Publishing Company. It is distributed under the terms of the Creative Commons Attribution 4.0 (CC-BY) License. Further distribution of this work is permitted, provided the original work is properly cited. 


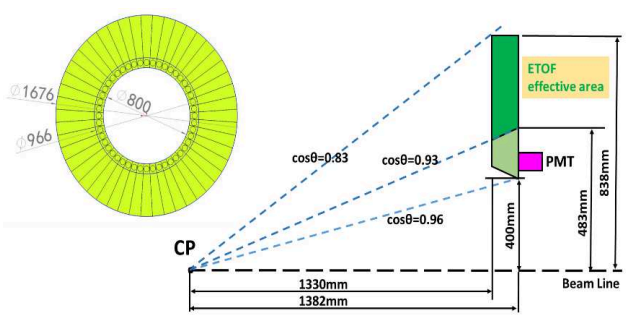

Fig. 1. Schematic drawing of ETOF location in BESIII spectrometer.

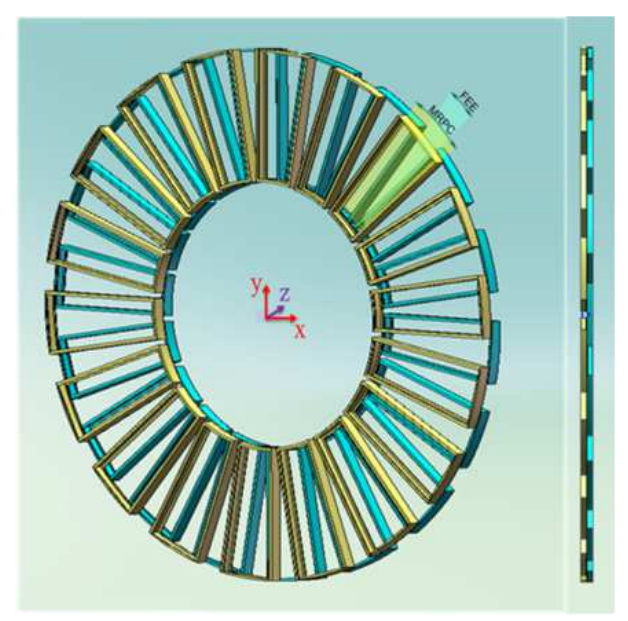

Fig. 2. The schematic drawing of MRPC ETOF upgrade at BESIII.

endcaps of the main drift chamber ${ }^{4}$. Another reason is the uncertainties of the particle positions hitting the scintillators that produce jittering for the time resolution in the process of position-time correction.

In the MRPCs, the gas gap between the electrodes is divided by means of internal plates which are physical barriers stopping the avalanche growing too big. It is possible to apply a very intense electric field and still operate the device in avalanche mode with many advantages in terms of time resolution and rate capability. It has been used successfully as TOF detectors in several experiments, such as ALICE at $\mathrm{LHC}^{5-6}$, STAR at RHIC ${ }^{7-8}$, with the time resolution of 80-90 ps.

To fully cover the BESIII endcap, the new ETOF is made of 72 fan shaped MRPCs and 36 on each end as shown in Fig. 2. Adjacent modules are staggered slightly to avoid dead regions. The effective areas of the MRPC rings have inner radius of $478 \mathrm{~mm}$ and outer radius $822 \mathrm{~mm}$. 


\section{The multi-gap resistive plate chamber}

The MRPCs for the BESIII ETOF are designed as double-stack strips. Floating glass sheets are used as the resistive plates. The thickness are $0.4 \mathrm{~mm}$ and $0.55 \mathrm{~mm}$ for the inner and outer glass, respectively. Each stack has six gaps of 220 um width made by fishing lines, the two stacks are placed on each side of a central anode. Two $3 \mathrm{~mm}$ thick honeycomb boards are fixed to the surface of the detector to avoid structural deformation. In the preliminary design two readout modes, namely singleend and double-end, are considered. After the beam test with several prototypes, the double-end readout MRPC is chosen for the better time resolution ${ }^{9-10}$.

There are quality control procedures in each step of the production chain. The quality control process includes the raw material performance such as geometry size, the glass surface quality and the bulk resistivity, the graphite painting quality and surface resistivity, the cleanness requirements, etc. MRPC assembling was carried out in a clean room. The temperature and moisture of the assembling workbench can be controlled and its cleanliness reaches $100 \mathrm{~K}$. The temperature is $22^{\circ} \mathrm{C} \pm 2^{\circ} \mathrm{C}$ and the moisture is maintained below 55\%. Several assembling tools are also designed to improve the production efficiency and ensure the quality of the products.

A dedicated cosmic ray test system was built in the University of Science and Technology of China to test the MRPCs during production process. The cosmic ray telescope was composed of two fan shaped scintillator counters, placed above and below the MRPC module separately. Each time three or four MRPCs were tested together. The high voltage was supplied by the CAEN N471A module, and the data acquisition system was based on commercial VME TDC modules.

After the mass production is finished, long-time cosmic ray tests in IHEP is performed in the lab as a final step of the Q\&A procedure. MRPC modules are stacked on four semicircle platforms. In order to trigger on the passing particles, 18 pairs of fan shaped scintillator counters are placed above and below the MRPC module separately. In this station the gas and HV system, the readout electronics, the data acquisition system for the new BESIII ETOF are used and tested. During the three months test, all the hardware run smoothly. Small numbers of dead channels due to no gas flow or HV supply are found and fixed. The intrinsic time resolution of MRPC is measured to be approximately $60 \mathrm{ps}$ and the average detection efficiency is above $96 \%^{11}$.

\section{ETOF subsystems}

The high voltage system of BESIII ETOF supplies positive voltage to the anodes $(+7 \mathrm{kV})$ and negative voltage to the cathodes $(-7 \mathrm{kV})$ independently. In the HV system, a NIM crate with nine HV modules was employed to supply 36 channels $\mathrm{HV}$ to the distributor, in which one HV input channel is divided into four output channels to the 72 modules. The HV control and monitoring system is incorporated into the BESIII slow control system. 
The gas system is made of 36 independent lines, 18 per end. Each line serves two modules serially. Flows of pure Freon, $\mathrm{S} F_{6}$ and iso- $C_{4} H_{10}$ are controlled by a MKS247B mass flow controller to form the working mixture. The flow rate is approximately two volume change per day. The gas mixture firstly fills the buffer tank, and then the gas flow is divided into 36 branches. The flow of each branch is controlled by a meter valve. The output gas from the MRPCs goes through bubblers and is vented to the atmosphere.

The readout electronics system consists of front end electronics (FEE) boards, Calibration-Threshold-Test-Power (CTTP) boards in NIM crate, Time-to- Digital (TDIG) converter modules, fast control modules and CLOCK modules in VME crate which works in pipeline mode with a clock frequency of about $40 \mathrm{MHz}$.

Each MRPC matches one FEE module with twenty four channels based on NINO ${ }^{12}$, which features time-over-threshold (TOT) measurement. The timing accuracy is better than 15 ps RMS for each channel when the input charge is larger than $100 \mathrm{fC}^{13}$. The FEE boards with connectors and cables are fixed on the surfaces of the aluminium boxes of the MRPC modules. Connectors (50 pin QSS-025-01-l-DRA-MTI) and shielded differential cables (SQCD-025) are used to connect the FEE and the TDIG ${ }^{14}$. The CTTP board provide power, threshold and test signal for the FEE. It also receives and transit the OR differential signals from the FEE to ETOF trigger subsystem.

The FEE signals are fed into TDIG modules, on each of which high performance TDCs ${ }^{15}$ are utilized for precise time measurements. The L1 trigger is sent via fast control module to TDIGs. The CLOCK module receives the synchronized clock from the master, and fans out clocks to feed modules inside the same VME crates.

The BESIII data acquisition system adopts the techniques of multi-level buffering, parallel processing, high-speed VME readout and network transmission. Also, the running status such as the event rate, noise level, the time spectrums and hitmap are monitored by a computer program to provide real-time information for the entire system.

\section{The installation and commissioning of MRPCs}

According to the project design, each MRPC module together with FEE will be mounted on the endcap of endcap EMC. If FEE or MRPC module is needed to repair, the endcap EMC should be firstly detached. The installation process lasts about two months, from August 1st to October 7th in 2015. Everyday four or six MRPCs can be installed, then the gas pipes and HV cables are connected. In the evening, the MRPCs installed previous day will be test with random triggers and the noise ratio of each channel should be less than $0.1 \%$.

The new ETOF commissioned together with BESIII since January 2016 and the status of all the hardware run smoothly. Physics data-taking begins after several days' debugging of ETOF trigger sub-system. After the calibration with Bhabha 

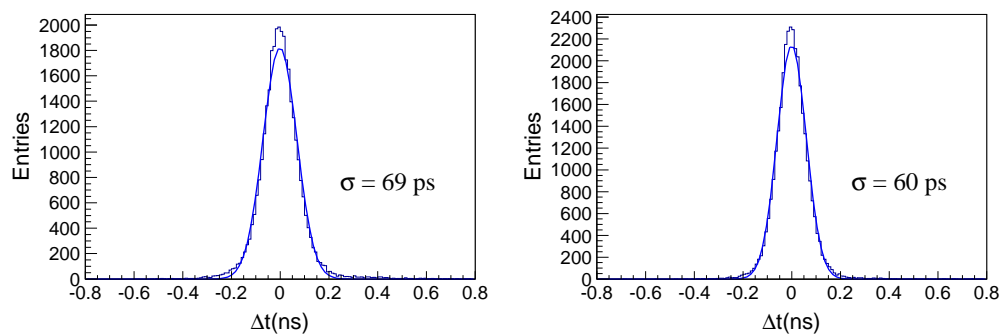

Fig. 3. (a) $t_{\text {meas }}-t_{\text {pred }}$ distribution of one-end of MRPCs; (b) $t_{\text {meas }}-t_{\text {pred }}$ distribution of two-ends of MRPCs.

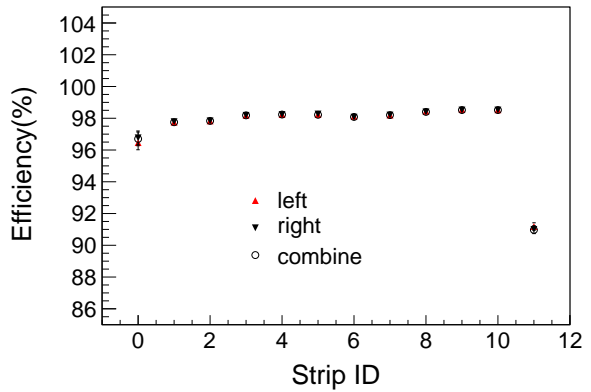

Fig. 4. Detection efficiency versus strip.

events, the very promising time resolutions of 69 and 60 ps for one-end and twoends of MRPCs are obtained(Fig. 3). The average detection efficiency of two-ends is about $97.5 \%$ (Fig. 4) ${ }^{16}$. For $800 \mathrm{MeV}$ Pion samples, the time resolution of two-ends is about $70 \mathrm{ps}$.

\section{Summary}

More than eighty bare MRPCs have been successfully produced to build the new BESIII ETOF. The subsystems of ETOF, such as the HV system, the gas control system were successfully controlled by the BESIII slow control system with a stable operation. The readout and the trigger electronics, online and offline software were tested together with the MRPCs. After the calibration with Bhabha events, the very promising time resolutions of 69 and 60 ps for one-end and two-ends of MRPCs are obtained. The average detection efficiency of two-ends is about $97.5 \%$. For 800 $\mathrm{MeV}$ Pion samples, the time resolution of two-ends is about 70ps. These results indicates the upgrade of BESIII ETOF is successful.

\section{References}

1. M. Ablikim et al., Design and construction of the BESIII detector, Nucl. Instrum. Meth. A 614 (2010) 345. 
2. C. Zhang for BEPC\&BEPCII Teams, Performance of the BEPC and progress of the BEPCII, Proceedings of APAC (2004) 15-19, Gyeongju, Korea.

3. C. Zhao et al., Time calibration for the end cap TOF system of BESIII, Chin. Phys. $C$ (HEPENP) 35 No.1, (2011) 72.

4. H. Zhang et al., A GEANT4 Simulation Studyof BESIII endcap TOF Upgrade, Chin. Phys. C 37, No. 9, (2013) 096002 .

5. D. De Gruttola et al., Study of the cosmic data taken with the ALICE TOF detector at the LHC, Nucl. Instrum. Meth. A 661 (2012) 102.

6. A. Alici, The MRPC-based ALICE time-of-flight detector: Status and performance, Nucl. Instrum. Meth. A 706 (2013) 29.

7. F. Geurts, M. Shao et al., Performance of the prototype MRPC detector for STAR, Nucl. Instrum. Meth. A $\mathbf{5 3 3}$ (2004) 60.

8. W.J. Llope, The large-area time-of-flight upgrade for STAR, Nucl. Instrum. Meth. B 241 (2005) 306.

9. Y. Sun et al., A prototype MRPC beam test for the BESIII ETOF upgrade, Chin. Phys. C (HEPENNP) 36 No.5, (2012) 429.

10. S. Yang et al., Test of high time resolution MRPC with different readout modes for the BESIII upgrade, Nucl. Instrum. Meth. A 763 (2014) 190.

11. Xiao-zhuang Wang et al., The cosmic ray test of MRPCs for the BESIII ETOF upgrade, Eur. Phys. J. C 76 (2016) 211.

12. F. Anghinolfi et al., NINO: an ultrafast low-power front-end amplifier discriminator for the time-of-flight detector in the ALICE experiment, Nucl. Instrum. Meth. $\mathbf{5 3 3}$ (2004) 183.

13. M. Despeisse et al., Low-Power Amplifier-Discriminators for High Time Resolution Detection, IEEE T. Nucl. Sci. 56 (2009) 375.

14. H. Fan et al., A High-Density Time-to-Digital Converter Prototype Module for BES III End-Cap TOF Upgrade, IEEE T. Nucl. Sci. 60 (2013) 3563.

15. M. Mota et al., A flexible multi-channel high-resolution time-to-digital converter ASIC, IEEE Nucl. Sci. Conf. R. Lyon, France, 15-20 Oct. 2000.

16. Z. Wu et al., First results of the new endcap TOF commissioning at BESIII, JINST 11 (2016) C07005. 